\title{
POLYMER GEOMETRY AT PLANCK SCALE AND QUANTUM EINSTEIN EQUATIONS
}

\author{
ABHAY ASHTEKAR \\ Center for Gravitational Physics and Geometry \\ Physics Department, Penn State, University Park, PA 16802
}

\begin{abstract}
Over the last two years, the canonical approach to quantum gravity based on connections and triads has been put on a firm mathematical footing through the development and application of a new functional calculus on the space of gauge equivalent connections. This calculus does not use any background fields (such as a metric) and is thus well-suited to a fully non-perturbative treatment of quantum gravity. Using this framework, quantum geometry is examined. Fundamental excitations turn out to be one-dimensional, rather like polymers. Geometrical observables such as areas of surfaces and volumes of regions have purely discrete spectra. Continuum picture arises only upon coarse graining of suitable semi-classical states. Next, regulated quantum diffeomorphism constraints can be imposed in an anomaly-free fashion and the space of solutions can be given a natural Hilbert space structure. Progress has also been made on the quantum Hamiltonian constraint in a number of directions. In particular, there is a recent approach based on a generalized Wick transformation which maps solutions to the Euclidean quantum constraints to those of the Lorentzian theory. These developments are summarized. Emphasis is on conveying the underlying ideas and overall pictures rather than technical details.
\end{abstract}

\section{Introduction}

It is well known that quantum general relativity is perturbatively non-renormalizable. Particle theorists often take this to be a sufficient reason to abandon general relativity and seek an alternative which has a better ultraviolet behavior in perturbation theory. However, one is by no means forced to this route. For, there do exist a number of field theories which are perturbatively non-renormalizable but are $e x$ actly soluble. An outstanding example is the Gross-Neveau model in 3 dimensions, $(G N)_{3}$, which was recently shown to be exactly soluble rigorously [1]. Furthermore, the model does not exhibit any mathematical pathologies. For example, it was at first conjectured that the Wightman functions of a non-renormalizable theory would have a worse mathematical behavior. The solution to $(G N)_{3}$ showed that this is not the case; as in familiar renormalizable theories, they are tempered distributions. Thus, one can argue that, from a structural viewpoint, perturbative renormalizability is a luxury even in Minkowskian quantum field theories. Of course, it serves as a powerful guiding principle for selecting physically interesting theories since it ensures that the predictions of the theory at a certain length scale are independent of the potential complications at much smaller scales. But it is not a consistency check on the mathematical viability of a theory. Furthermore, in quantum gravity, 
one is interested precisely in the physics of the Planck scale; the short-distance complications are now the issues of primary interest. Therefore, it seems inappropriate to elevate perturbative renormalizability to a viability criterion.

Even if one accepts this premise, however, one is led to ask: Are there specific reasons that suggest that quantum general relativity may exist at a non-perturbative level? The answer, I believe, is in the affirmative. There are growing indications from a number of different directions -computer simulations, canonical quantization and string theory - that the quantum geometry of space-time would be quite different from classical geometry $[2,4]$. Specifically, in this review we will see that, in a rather wide class of non-perturbative quantum gravity theories, the fundamental excitations of the gravitational field are one dimensional rather than three; they resemble onedimensional networks rather than three dimensional waves. At the Planck scale, geometry has a close similarity with polymers and the three dimensional continuum arises only as a "coarse-grained" approximation. Perturbative treatments, on the other hand, assume the validity of a continuum picture at all scales. The ultraviolet problems one encounters may simply be a consequence of the fact that the true microscopic structure of space-time is captured so poorly in these treatments. Put differently, if the continuum picture is replaced by a more faithful one, the "effective dimension" of space-time could be smaller than four, whence the theory could have a much better behavior non-perturbatively.

In this article, I will accept this premise and consider a non-perturbative quantization of general relativity. Our approach will differ from the traditional methods of constructing quantum field theories in a number of ways. First, we will not use any background metric or connection or indeed any background field. In this sense all our constructions will respect the underlying diffeomorphism invariance of the theory. Second, we will always work in the continuum. For actual computations e.g., of the spectra of interesting operators, we will often introduce "floating lattices" or graphs. But these will only serve as computational devices in an already defined continuum theory. Third, in the main constructions, we will refer only to the Planck scale; a "macroscopic" scale will not feature in the regularization of operators or construction of the theory itself ${ }^{1}$.In particular, there will be no cut-offs at a fundamental level.

In the light of the text-book treatments of (flat space) quantum field theories, these features may seem surprising. Indeed, if we do not follow one of these standard methods, what then is our strategy? In general terms, the idea is to first re-examine the quantization problem from a somewhat broader perspective. One constructs a suitable algebra of functions on the classical phase space, promotes it to an operator algebra and then seeks its representations by operators on a Hilbert space. That is, one goes to the "root" of the quantization problem. Due to the presence of an infi-

1 Such scales may be needed to probe the physical meaning of the theory and will then arise from specific physical problems. For instance, one can seek semi-classical quantum states that, when coarse-grained on, say, the weak interaction scale, reproduce classical geometries. The problem then naturally provides the scale of $\approx 10^{-17} \mathrm{~cm}$. In the basic theory itself, these "macroscopic" scales do not feature. 
nite number of degrees of freedom, this problem is difficult in any field theory. For flat space field theories, the standard treatments provide strategies to attack this problem using the powerful machinery associated with the renormalization group. In the present context, where is there is no background metric (or any other field), at least at first sight, these strategies seem not to be as well-suited. What we need is a new functional calculus that respects the diffeomorphism invariance of the underlying theory. Our strategy is to first develop such a calculus and then use it to construct the Hilbert spaces of states and to regularize physically interesting operators thereon, directly in the continuum. The resulting Hilbert spaces and operators are somewhat unconventional. In particular, the fundamental excitations can not be identified with gravitons. To particle physicists, this may seem surprising at first. However, on further thought, one realizes that this is precisely what one should expect: after all gravitons are spin-2 excitations on a Minkowskian background and should therefore arise only as approximate notions in any fully non-perturbative treatment. There already exist detailed results which indicate how this can come about [5]. However the relation between our approach and the standard constructions based on the renormalization group techniques remains unclear; it would be extremely interesting to investigate this issue in detail.

The general approach followed in this review is being pursued by a large number of researchers in about a dozen different groups. I will not attempt to present a comprehensive or even a systematic survey. Rather, I will focus only on some of the recent mathematical developments. These were motivated in part by earlier exploratory work by a number of colleagues, especially Jacobson, Rovelli and Smolin $[6,7]$. For brevity, however, I will forego motivational remarks and attempt to present the final picture in a concise fashion focusing on the recent mathematical developments. (For reviews of the earlier work, see [8-11].)

\section{Difficulties and Strategies}

The non-perturbative approach I wish to discuss is based on canonical quantization. The canonical formulation of general relativity was first obtained in the late fifties and early sixties through a series of papers by Bergmann, Dirac and Arnowitt, Deser and Misner. In this formulation, general relativity arises as a dynamical theory of 3-metrics. The framework was therefore named geometrodynamics by Wheeler and used as a basis for canonical quantization both by him and his associates and by Bergmann and his collaborators. The framework of geometrodynamics has the advantage that classical relativists have a great deal of geometrical intuition and physical insight into the nature of the basic variables -3-metrics $g_{a b}$ and extrinsic curvatures $K_{a b}$. For these reasons, the framework has played a dominant role, e.g., in numerical relativity. However, it also has two important drawbacks. First, it sets the mathematical treatment of general relativity quite far from that of theories of other interactions where the basic dynamical variables are connections rather than metrics. Second, the equations of the theory are rather complicated in terms of metrics and extrinsic curvatures; being non-polynomial, they are difficult to carry 
over to quantum theory with a reasonable degree of mathematical precision.

For example, consider the standard Wheeler-DeWitt equation:

$$
\left[\sqrt{\frac{G^{2} \hbar^{2}}{g}}\left(g^{a b} g^{c d}-\frac{1}{2} g^{a c} g^{b d}\right) \frac{\delta}{\delta g_{a c}} \frac{\delta}{\delta g_{b d}}-\sqrt{\frac{g}{G^{2} \hbar^{2}}} R(g)\right] \circ \Psi(g)=0,
$$

where $g$ is the determinant of the 3-metric $g_{a b}$ and $R$ its scalar curvature. As is often emphasized, since the kinetic term involves products of functional derivatives evaluated at the same point, it is ill-defined. However, there are also other, deeper problems. These arise because, in field theory, the quantum configuration space -the domain space of wave functions $\Psi$ - is larger than the classical configuration space. While we can restrict ourselves to suitably smooth fields in the classical theory, in quantum field theory, we are forced to allow distributional field configurations. Indeed, even in the free field theories in Minkowski space, the Gaussian measure that provides the inner product is concentrated on genuine distributions. This is the reason why in quantum theory fields arise as operator-valued distributions. One would expect that the situation would be at least as bad in quantum gravity. If so, even the products of the 3-metrics that appear in front of the momenta and the meaning of the scalar curvature in the potential term are obscure. The left hand side of the Wheeler-DeWitt equation is seriously ill-defined and must be regularized appropriately.

However, as I just said, the problem of distributional configurations arises already in the free field theory in Minkowski space-time. There, we do know how to regularize physically interesting operators. So, why can we not just apply those techniques in the present context? The problem is that those techniques are tied to the presence of a background Minkowski metric. The covariance of the Gaussian measure, for example, is constructed from the Laplacian operator on a space-like plane defined by the induced metric and normal ordering and point-splitting regularizations also make use of the background geometry. In the present case, we do not have background fields at our disposal. We therefore need to find another avenue. What is needed is a suitable functional calculus -integral and differentialthat respects the diffeomorphism invariance of the theory.

What space are we to develop this functional calculus on? Recall first that, in the canonical approach to diffeomorphism invariant theories such as general relativity or supergravity, the key mathematical problem is that of formulating and solving the quantum constraints. (In Minkowskian quantum field theories, the analogous problem is that of defining the regularized quantum Hamiltonian operator.) It is therefore natural to work with variables which, in the classical theory, simplify the form of the constraints. It turns out that, from this perspective, connections are better suited than metrics [12].

To see this, recall first that in geometrodynamics we can choose as our canonical pair, the fields $\left(E_{i}^{a}, G^{-1} K_{a}^{i}\right)$ where $E_{i}^{a}$ is a triad (with density weight one) and $K_{a}^{i}$, the extrinsic curvature. Here $a$ refers to the tangent space of the 3-manifold and $i$ is the internal $S O(3)$-or, $S U(2)$, if we wish to consider spinorial matter- index. The triad is the square-root of the metric in the sense that $E_{i}^{a} E^{b i}=: g g^{a b}$, where $g$ 
is the determinant of the covariant 3-metric $g_{a b}$, and $K_{a}^{i}$ is related to the extrinsic curvature $K_{a b}$ via: $K_{a}^{i}=(1 / \sqrt{g}) K_{a b} E^{b i}$. Let us make a (real) transformation:

$$
\left(E_{i}^{a}, G^{-1} K_{a}^{i}\right) \mapsto\left(A_{a}^{i}:=G^{-1}\left(\Gamma_{a}^{i}-K_{a}^{i},\right) E_{i}^{a}\right),
$$

where $\Gamma_{a}^{i}$ is the spin connection determined by the triad. It is not difficult to check that this is a canonical transformation on the real phase space $[12,13]$. It will be convenient to regard $A_{a}^{i}$ as the configuration variable and $E_{i}^{a}$ as the conjugate momentum so that the phase space has the same structure as in the $S U(2)$ YangMills theory. Let us begin by simply writing down the simplest equations we can, without any reference to a background field, using these variables. They are:

$$
\begin{aligned}
\mathcal{G}_{i} & :=D_{a} E_{i}^{a}=0 \\
\mathcal{V}_{b} & :=E_{i}^{a} F_{a b}^{i} \equiv \operatorname{Tr} E \times B=0 \\
\mathcal{S} & :=\epsilon^{i j k} E_{i}^{a} E_{j}^{b} F_{a b k} \equiv \operatorname{Tr} E \cdot E \times B=0
\end{aligned}
$$

These are the "simplest" equations in the following sense: Among non-trivial gauge covariant expressions, the left side of the first equation is the only one which is at most linear in $E$ and $A$; that of the second is the only one that is at most linear in $E$ and quadratic in $A$; and, that of the third is the only one that is at most quadratic in each of $E$ and $A$. (There is no gauge covariant expression which is linear in $A$ and at most quadratic in E.) Somewhat surprisingly, the first two equations provide us precisely with the 6 of 7 constraints of general relativity: the Gauss constraint $\mathcal{G}_{i}$ which generates internal frame rotations and the vector constraint $\mathcal{V}_{b}$ that generates spatial diffeomorphisms [12]. The third equation is almost -but not quite- the last (scalar or Hamiltonian) constraint we seek. To see this, let us translate it in to geometrodynamical variables. It then reduces to the familiar Euclidean Hamiltonian constraint [13]

$$
R+K^{a b} K_{a b}-K^{2}=0,
$$

rather than the Lorentzian Hamiltonian constraint

$$
R-K^{a b} K_{a b}+K^{2}=0,
$$

which, as one might expect, carries opposite signs in front of terms which are quadratic in momenta. (Intuitively, the "Wick" rotation should map the momentum to $i$ times the momentum.) The Gauss and the vector constraints are insensitive to the signature.

To get the Lorentzian theory, the most straightforward avenue is to consider complex connections ${ }^{c} A_{a}{ }^{i}:=\Gamma_{a}^{i}-i K_{a}^{i}$. This avenue has been pursued vigorously in the literature [8-11]. In this article, however, I will present another possibility which has arisen from a recent idea of Thomas Thiemann's and which may allow one to pass from the solutions to the Euclidean quantum constraints to the Lorentzian ones via a generalized Wick transformation. This strategy will be discussed in Section 5 . For the moment, let us note only that $S U(2)$ connections can be regarded as the configuration variables also in general relativity. Thus, the classical configuration 
space is $\mathcal{A} / \mathcal{G}$, the space of suitably smooth connections modulo gauge transformations. Our task is to find the corresponding quantum configuration space and to develop functional calculus on it.

I will conclude this discussion with a curiosity: The Euclidean signature seems to be easier to handle in other approaches as well, in particular, Connes' noncommutative geometry and string theory. In the Connes' "geometrization" of the standard model, one is naturally led to work with the Euclidean signature and the extension of the framework to the Lorentzian regime is still to be worked out. In string theory, while one can allow the target space geometry to be Lorentzian, in all the discussions that I am aware of, the world sheet is kept Euclidean. In perturbation theory off Minkowskian backgrounds, as far as the S-matrix theory is concerned, this seems like an obvious extension of the strategy one adopts in field theory. However, in non-perturbative contexts, such as the discussion of mirror symmetry, restriction to Riemannian world-sheets appears artificial from a physical perspective. As in the above discussion of the Hamiltonian constraint, in both these examples, one needs an appropriate, generalized Wick transform.

\section{Tools}

The classical configuration space $\mathcal{A} / \mathcal{G}$ is the quotient of the space $\mathcal{A}$ of suitably smooth connections by the group $\mathcal{G}$ of local gauge transformations. $\mathcal{A} / \mathcal{G}$ can be endowed with topology in a standard way, using a Sobolev norm on the space $\mathcal{A}$. The key question now is: What is the quantum configuration space? More precisely, in the connection representation, what is the domain space of quantum wave functions? In quantum mechanics -i.e., in the quantum theory of systems with a finite number of degrees of freedom- the classical and the quantum configuration spaces agree. In field theory, as noted in Section 1, the quantum configuration space is a substantial enlargement of the classical one; in scalar field theories, for example, although the classical configurations are smooth (say $C^{2}$ ) functions on a $t=$ const slice, the quantum configuration space consists of all tempered distributions. Furthermore, this enlargement is not a mere technicality: the set of smooth configurations is of zero measure with respect to the Gaussian measure that determines the inner product! The regularization problems of quantum field theory can be traced back to this fact.

The text-book treatments for the construction of quantum configuration spaces are not directly applicable in the present case because they are geared to the case in which classical configuration spaces are linear. Nonetheless, a natural strategy is available [14]. Recall first that the Wilson-loop functions, $T_{\alpha}(A):=\frac{1}{2} \operatorname{Tr} \mathcal{P} \exp \oint A$.dl, form a natural (over)complete set of functions on $\mathcal{A} / \mathcal{G}$ in the sense that they suffice to separate the points of $\mathcal{A} / \mathcal{G}$. (Trace is taken in the fundamental representation.) They are thus natural candidates for our configuration variables ${ }^{2}$. It is straightforward

\footnotetext{
2 All loops will be based at some arbitrarily chosen but fixed point on $\Sigma$. For technical reasons, we will restrict ourselves to piecewise analytic loops. Some of the key results have been extended to the smooth
} 
to construct a $C^{\star}$-algebra generated by these $T_{\alpha}$. It is called the holonomy algebra and denoted by $\overline{\mathcal{H A}}$. The first step in the quantization procedure is to develop the representation theory of $\overline{\mathcal{H}} \mathcal{A}$. A natural candidate for the quantum configuration space will arise from this theory.

Since elements of this algebra are all configuration variables, the algebra is Abelian and it is equipped with an identity, the Wilson loop function associated with the trivial (point) loop. Now, the representation theory of Abelian $C^{\star}$-algebras with identity has been developed in detail by Gel'fand and Naimark. One of their basic results is that any such $C^{\star}$-algebra is naturally isomorphic with the $C^{\star}$-algebra of all continuous functions on a compact, Hausdorff space, called the spectrum of the algebra. Thus, every element $a$ of the algebra is canonically represented by a concrete function $\check{a}$ on the spectrum. In our case, the algebra $\overline{\mathcal{H A}}$ is smaller than the algebra of all bounded continuous functions on $\mathcal{A} / \mathcal{G}$ and the spectrum turns out to be larger than $\mathcal{A} / \mathcal{G}$. However, since the elements of $\overline{\mathcal{H} \mathcal{A}}$ suffice to separate points of $\mathcal{A} / \mathcal{G}$, it follows that $\mathcal{A} / \mathcal{G}$ is densely embedded in the spectrum [16]. Thus, we can regard the spectrum as a completion of $\mathcal{A} / \mathcal{G}$. To emphasize this point, we will denote it by $\overline{\mathcal{A} / \mathcal{G}}$.

Since $\overline{\mathcal{A} / \mathcal{G}}$ is a compact Hausdorff space, using the Riesz representation theorem, one can conclude that every cyclic representation of $\overline{\mathcal{H A}}$ by bounded operators on a Hilbert space is of the following type: The Hilbert space is $L^{2}(\overline{\mathcal{A} / \mathcal{G}}, d \mu)$ for some measure $\mu$ and the operators $\hat{T}_{\alpha}$ act via:

$$
\left(\hat{T}_{\alpha} \circ \Psi\right)(\bar{A})=\check{T}_{\alpha}(\bar{A}) \Psi(\bar{A}),
$$

where the function $\check{T}$ on $\overline{\mathcal{A} / \mathcal{G}}$ is the Gel'fand transform of $T_{\alpha}$. Thus, $\overline{\mathcal{A} / \mathcal{G}}$ serves as an universal domain space for quantum states; it is therefore the quantum configuration space.

The details of this construction will not be needed in what follows. One just needs to note that a natural completion $\overline{\mathcal{A} / \mathcal{G}}$ of $\mathcal{A} / \mathcal{G}$ will serve as the quantum configuration space and that, even though $\mathcal{A} / \mathcal{G}$ is a rather complicated, infinite dimensional space, $\overline{\mathcal{A} / \mathcal{G}}$ is compact.

Our task then is to develop integral and differential calculus on $\overline{\mathcal{A} / \mathcal{G}}$ : measures and integration theory are needed to specify the inner-product and differential operators are needed to define observables. The task has been carried out in a series of papers [17-24]. In essence, this development was possible because there are three distinct ways of characterizing $\overline{\mathcal{A} / \mathcal{G}}$, each illuminating a specific aspect of its structure: first, as the Gel'fand spectrum of the holonomy algebra [14]; second, as the space of homomorphisms from the so-called hoop group ${ }^{3}$ to the gauge group $S U(2)$ [17]; and third, as a projective limit of configuration spaces associated with graphs

category by Baez and Sawin [15].

3 The hoop group $\mathcal{H G}$ of the 3-manifold $\Sigma$ is defined as follows. Consider the space of piecewise analytic, closed loops in $\Sigma$ which begin and end at the fixed point $x$. Regard two loops as equivalent if the holonomy of any (smooth) $S U(2)$ connection around one equals that around the other. The space of equivalence classes then has a natural group structure, groups corresponding to different base points being isomorphic. 
in $\Sigma[19,20]$. As a result, $\overline{\mathcal{A} / \mathcal{G}}$ has structure which is much richer than what one might expect at first.

That integral calculus can be developed [17-20] is perhaps not surprising: after all, $\overline{\mathcal{A} / \mathcal{G}}$ is a compact, Hausdorff space and therefore admits regular measures. The development of a differential calculus, on the other hand, seems hopeless at first since Gel'fand spectra are only topological spaces; the Gel'fand theory does not endow them with a differential structure. In particular, it seems difficult to convert $\overline{\mathcal{A} / \mathcal{G}}$ into an infinite dimensional manifold. However, because our $C^{\star}$-algebra is special -the holonomies have a natural geometrical meaning- $\overline{\mathcal{A} / \mathcal{G}}$ can be regarded as the projective limit of finite dimensional manifolds (the third characterization above). This enables one to introduce the notion of smooth functions on $\overline{\mathcal{A} / \mathcal{G}}$ and use it to introduce forms, vector fields, and a number of differential and integral operators without any reference to a background metric on the underlying 3-manifold $\Sigma$ [21]. We will see some illustrations of how this is achieved in the next section.

To proceed further in a concrete fashion, one has to choose a specific measure on $\overline{\mathcal{A} / \mathcal{G}}$ and work in the representation of the holonomy algebra it provides. The algebra itself imposes no restriction on this choice; for any regular measure $\mu$, the Wilson loop operators $\hat{T}_{\alpha}$ are bounded self-adjoint operators on the resulting Hilbert space $H \equiv L^{2}(\overline{\mathcal{A} / \mathcal{G}}, d \mu)$. It is the requirement that the momentum operators be self-adjoint that restricts the measure. (A similar situation occurs already in non-relativistic quantum mechanics: while the position operator $\hat{X}$ is self-adjoint on $L^{2}(R, f d x)$ for any (regular) function $f$, the momentum operator $\hat{P} \equiv-i \hbar d / d x$ is self adjoint only if $f$ is a constant. Thus, it is the self-adjointness of the explicitly defined momentum operators that singles out the Lebesgue measure $d x$.) In our case, the momenta are essentially the electric fields. However, electric fields are not gauge invariant and to obtain an operator which descends to $\mathcal{A} / \mathcal{G}$-i.e., has a meaningful action on the space of gauge invariant wave functions- further work is needed ${ }^{4}$. When this is done, one obtains momentum operators that can act on suitably regular functions $\Psi(\bar{A})$ on $\overline{\mathcal{A} / \mathcal{G}}$. The requirement that these momentum operators be self-adjoint then picks out a measure $\mu_{o}$ on $\overline{\mathcal{A} / \mathcal{G}}[23]$.

This measure has four important properties [17-19]: i) it is normalized; the total $\mu_{o}$ measure of $\overline{\mathcal{A} / \mathcal{G}}$ is 1 ; ii) it is faithful; the integral of every non-negative, continuous function on $\overline{\mathcal{A} / \mathcal{G}}$ is non-negative and vanishes if and only if the function is identically

This group is $\mathcal{H G}$. While the definition refers to a specific gauge group, the resulting $\mathcal{H G}$ turns out to be largely independent of this choice: there are only two hoop groups; one Abelian (associated with Abelian gauge groups) and the other non-Abelian [17].

4 The simplest strategy is to use 2-dimensional strips $S$, parametrized by $(\sigma, \tau)$, which are foliated by closed loops $\tau=$ const., and consider the associated "momentum variables" $P_{S}(A, E):=\int d S^{a b} \epsilon_{a b c^{-}}$ $\left(\operatorname{Tr} E^{c}(\sigma, \tau) U(\sigma, \tau)\right)$, where $U$ is the holonomy of $A$ around the loop $\tau=$ const, evaluated at the point $(\sigma, \tau)$ of $S$. This is a gauge invariant function on the phase space, linear in the momentum $E$, and depends only on the foliation of $S$ (i.e., not on the details of the parameterization). Since there are no background fields, the Poisson algebra of configuration and momentum variables, $T_{\alpha}$ and $P_{S}$, can be expressed in an elegant fashion, using only the topological properties of loops and strips in 3-dimensions [25]. 
zero; iii) $\mu_{o}$ is invariant under the (induced action on $\overline{\mathcal{A} / \mathcal{G}}$ of the) diffeomorphism group on the underlying 3-manifold $\Sigma$; and, iv) $\mu_{o}$ is concentrated on genuinely generalized connections; the $\mu_{o}$-measure of the classical configuration space $\mathcal{A} / \mathcal{G}$ is zero! The last result is the analog of the standard situation in flat space quantum field theory referred to above; the classical configuration space is topologically dense in the quantum configuration space but measure theoretically sparse.

We can use this measure to construct a Hilbert space $H_{o}:=L^{2}\left(\overline{\mathcal{A} / \mathcal{G}}, d \mu_{o}\right)$. This is the space of kinematic states of quantum gravity. It is "kinematic" because we are yet to impose quantum constraints to select physical states which have dynamical information; it is the quantum analog of the full phase space of general relativity. To solve quantum dynamics, we have to first regularize quantum constraints and express them as well-defined operators on $H_{o}$ and then isolate the kernel of these operators. We will turn to this task in Section 5.

We will conclude this section by pointing out two properties of $H_{o}$ which will play an important role in the subsequent discussion.

First, $H_{o}$ admits [24] an interesting orthonormal basis, obtained by a generalization of Penrose's spin networks [27] (which, although introduced in quite a different context, were also motivated by quantum gravity considerations. See also [26].). A penrose network is a closed graph, each of whose vertices is trivalent (i.e., has three edges), with an assignment of representation of SU(2) (i.e., of a half-integral number $j$ ) to each of its edges, such that, if $j_{1}, j_{2}, j_{3}$ label edges incident at any one vertex, we must have $\left|j_{1}-j_{2}\right| \leq j_{3} \leq j_{1}+j_{2}$ (and permutations thereof). Now, each such network $N$ defines a function $\Psi_{N}$ on $\overline{\mathcal{A} / \mathcal{G}}$ as follows. First, one can show $[18,21]$ that every (generalized) connection $\bar{A}$ assigns to each edge an element of $S U(2)$, the holonomy. Using this assignment, we can associate a matrix with each edge in the Penrose network. The constraint on the choice of representations ensures that all the resulting matrices can be consistently contracted to produce a number, the value of $\Psi_{N}$ on the chosen point $\bar{A}$ of $\overline{\mathcal{A} / \mathcal{G}}$. Remarkably, these states are orthonormal in $H_{o}$. However, they are not complete. To obtain a complete set, we have to allow higher valent networks -i.e., the ones which have an arbitrary (but finite) number of edges at any one vertex- assign to each edge a representation of $S U(2)$ and to each vertex a suitable contractor (or, an inter-twiner). The resulting space of states can then be used to obtain an orthonormal decomposition of $H_{o}$ into finite dimensional subspaces, each labelled by the network (embedded in $\Sigma$ ) and the assignment of representations $[24,23]$. (The dimensionality of each subspace is given by the number of independent contractors and one can introduce an orthonormal basis in each subspace using the Schmidt procedure.)

The second property of $H_{o}$ is that it admits an interesting dense subspace. Fix a closed graph $\gamma$ (i.e., a collection of vertices joined by analytic edges such that every vertex has at least two edges) in $\Sigma$. Every (generalized) connection $\bar{A}$ associates to each edge $e_{i}$ of $\gamma$ an element $g_{i}$ of $S U(2)$. Thus, there is a natural projection from $\overline{\mathcal{A} / \mathcal{G}}$ to $[S U(2)]^{n}$, where $n$ is the total number of edges in the graph. Using this projection, 
we can pull-back to $\overline{\mathcal{A} / \mathcal{G}}$ functions on $[S U(2)]^{n}$. These pull-backs,

$$
\Psi_{\gamma}(\bar{A}):=\psi\left(g_{1}(\bar{A}), \ldots, g_{n}(\bar{A})\right)
$$

for some smooth function $\psi$ on $[S U(2)]^{n}$ are called cylindrical functions on $\overline{\mathcal{A} / \mathcal{G}}$. Note that the functions $\Psi_{\gamma}$ only know about what the connection $\bar{A}$ is doing at points of $\Sigma$ which lie in the graph $\gamma$. Yet, as we vary the graph and consider more and more vertices and edges, we obtain a bigger and bigger collection of functions on $\overline{\mathcal{A} / \mathcal{G}}$. They are all square-integrable with respect to $\mu_{o}$ and, furthermore, span a dense subspace of $H_{o}$. This space $\operatorname{Cyl}(\overline{\mathcal{A} / \mathcal{G}})$ of all cylindrical functions plays an important role in calculations, rather analogous to that played by the space $C_{o}^{\infty}(R)$ of smooth functions of compact support in quantum mechanics on a line. Just as we often first define operators on $C_{o}^{\infty}(R)$ and then extend them to (other, better suited dense subspaces of $L^{2}(R, d x)$, in the present context, we will often define operators first on $\operatorname{Cyl}(\overline{\mathcal{A} / \mathcal{G}})$ and then extend them.

These two properties of $H_{o}$ provide considerable intuition about the nature of quantum states that we have been led to consider. Since an orthonormal basis is provided by networks, it follows that the "elementary excitations" are 1-dimensional rather than three. They are "loopy" rather than "wavy". A typical excited state looks like a polymer rather than a smooth undulation on flat space. Just as a polymer in a sufficiently complex configuration behaves as if it were a 3-dimensional entity, we will see that the 1-dimensional excitations, if packed densely and superposed coherently, can approximate a 3-dimensional continuum geometry very well. The quantum states we have encountered here are qualitatively different from the ones we come across in Minkowskian quantum field theories. The main reason is the underlying diffeomorphism invariance: In absence of a background geometry it is not possible to introduce the familiar Gaussian measures and associated Fock spaces.

\section{Quantum Geometry}

The framework constructed in Section 3 provides us with sufficient tools to explore the nature of quantum geometry and, as we will see, the results are quite surprising.

Let us first recall that, in classical general relativity, geometrical observables can be regarded as functions on the phase space. Let us, for example, fix a smooth 2 -surface $S_{o}$ or a 3-dimensional region $R_{o}$ in the 3-manifold $\Sigma$. Then, given any triad field $E_{i}^{a}$, we can assign to $S_{o}$ an area, $A\left(S_{o}\right)$, and to $R_{o}$ a volume, $V\left(R_{o}\right)$ :

$$
A\left(S_{o}\right):=\int_{S_{o}}\left|E_{i}^{3} E^{3 i}\right|^{\frac{1}{2}} d^{2} x ; \quad \text { and } \quad V\left(R_{o}\right):=\int_{R_{o}}|\operatorname{det} E|^{\frac{1}{2}} d^{3} x,
$$

where, to simplify the presentation, we have chosen coordinates so that $S_{o}$ is given by $X_{3}=$ const. Thus, $A\left(S_{o}\right)$ and $V\left(R_{o}\right)$ can be regarded as functions on the full classical phase space (which happen to depend only on the triad, i.e. happen to be 
independent of the connection). Our problem now is to promote these functions to operators on the Hilbert space $H_{o}$-the quantum analog of the full phase space- and study their properties.

At first sight, the challenge seems unsurmountable. The first obstacle is that even the classical expressions are non-polynomial in the triads. Second, our wave functions $\Psi(\bar{A})$ have support on generalized connections which have a "distributional character." Hence, the task of regularizing formal expressions like

$$
\left|-\left(\frac{\delta}{\delta A_{3}^{i}(x)}\right)\left(\frac{\delta}{\delta A_{3}^{i}(x)}\right)\right|^{\frac{1}{2}}
$$

seems formidable. In addition, we do not have a background metric to simplify this task; we thus have the constraint that the final, regularized operators should not depend on any background fields. It turns out, however, that, using the functional calculus on $\overline{\mathcal{A} / \mathcal{G}}$, all these difficulties can be overcome $[28,23]$. (For an approach based on the loop representation, see [29] and, on lattice regularization, see [30]. I should add however that I do not yet know the precise relation to these approaches.)

One begins with the classical expressions, introduces a chart and point splits the triad fields using regulators, i.e., 2-point functions $f_{\epsilon}(x, y)$ which tend to $\delta^{3}(x, y)$ in the limit as $\epsilon$ tends to zero. One then notices that the resulting expressions can be promoted to operators which have a well-defined action on cylindrical functions on $\overline{\mathcal{A} / \mathcal{G}}$. One evaluates this action and, in the final expression, takes the limit $\epsilon \mapsto 0$. The limits are well-defined and yield operators which carry no memory of the background chart or regulators used in the procedure. They map cylindrical functions based on a graph $\gamma$ to cylindrical functions based on the same graph. Let us fix a graph with $\mathrm{n}$ edges. Then, given a cylindrical function $\Psi_{\gamma}(\bar{A})$ which is the pull-back of a function $\psi\left(g_{1}, \ldots, g_{n}\right)$ of $[S U(2)]^{n}$, the area operator yields:

$$
\hat{A}\left(S_{o}\right) \circ \Psi(\bar{A}):=\ell_{P}^{2} \sum_{\alpha}\left|\sum_{I_{\alpha} J_{\alpha}} K\left(I_{\alpha}, J_{\alpha}\right) X_{I_{\alpha}}^{i} X_{J_{\alpha}}^{i}\right|^{\frac{1}{2}} \circ \psi\left(g_{1}, \ldots, g_{n}\right) .
$$

Here $\ell_{P}$ is the Planck length; $\alpha$ ranges over the vertices of the graph $\gamma$ which intersect $S_{o} ; I_{\alpha}$ and $J_{\alpha}$ denote edges passing through the vertex $\alpha ; K\left(I_{\alpha}, J_{\alpha}\right)$ takes values $0, \pm 1$ depending on the orientation of the two edges relative to $S_{o}$; and, $X_{I}^{i}$ is the $i$-th right (left) invariant vector field on the $I_{\alpha}$-th copy of the gauge group if the edge $I_{\alpha}$ is outgoing (incoming) at the vertex $\alpha$. (Note that since there are three right (left) invariant vector fields on $S U(2)$, the index $i$ runs over 1,2,3.) Each vector field $X$ acts on only one of the arguments in the wave function, i.e., on the copy of the gauge group picked by the associated edge. Similarly, the volume operator is given by:

$$
\hat{V}\left(R_{o}\right) \circ \Psi(\bar{A}):=\ell_{P}^{3} \sum_{\alpha}\left|\sum_{I_{\alpha}, J_{\alpha}, K_{\alpha}} i \epsilon^{i j k} \epsilon\left(I_{\alpha}, J_{\alpha}, K_{\alpha}\right) X_{I_{\alpha}}^{i} X_{J_{\alpha}}^{j} X_{K_{\alpha}}^{k}\right|^{\frac{1}{2}} \circ \psi\left(g_{1}, \ldots, g_{n}\right),
$$

where the first sum now is over vertices which lie in the region $\mathrm{R}$ and $\epsilon\left(I_{\alpha}, J_{\alpha}, K_{\alpha}\right)$ is 0 if the three edges are linearly dependent at the vertex $\alpha$ and otherwise \pm 1 depending 
on the orientation they define. Thus, the two operators are well-defined on the space $\operatorname{Cyl}(\overline{\mathcal{A} / \mathcal{G}})$ of cylindrical functions on $\overline{\mathcal{A} / \mathcal{G}}$. Recall, however, that this space is dense in $H_{o}$. Using this fact and the properties of right and left invariant vector fields on $S U(2)$, one can show that they admit unique self-adjoint extensions on $H_{o}$.

For our purposes here, the details of these operators are not important. It would be sufficient to note just that closed form expressions of the fully regulated operators are available and they involve the actions of right and left invariant vector fields on the appropriate copies of $S U(2)$. Because these actions are completely understood, in practice, it is rather straightforward to compute how these operators act on cylindrical states associated with specific graphs.

A number of remarks are in order.

1. Given that the expressions of the classical observables are already rather involved, why are the expressions of the quantum operators relatively simple? The main reason is that the requirement of diffeomorphism invariance -i.e., absence of background fields - severely restricts the possible operators. Consider, for example, the area operator. Given a graph and a surface, the obvious diffeomorphism invariant notion is that of an intersection of the graph with the surface. Not surprisingly, the expression of the operator is a sum over intersections. At each intersection, the graph has a number of edges. So, all that the operator can do is to act on the copies of groups associated with these edges. That is, thanks to diffeomorphism invariance, the action of operators is reduced to simple algebraic operations in the representation theory of $S U(2)$ !

2. Since the two operators are self-adjoint, one can compute their spectra. They are purely discrete! Each of the Penrose spin network state is an eigenstate of both the operators. The area operators reduce to a sum of $S U(2)$-Laplacians, each of which has the familiar eigenvalues $\sqrt{j(j+1)}$. The eigenvalue of the volume operator on these states is, however, zero [30, 28]. Thus, higher valent spin networks are essential. Both operators again leave the (finite dimensional) Hilbert space associated with any of these networks invariant. They can therefore be diagonalized separately on each network. (Already in the 4-valent case, there exist states with non-zero volume.) This overall picture shows that the microscopic geometry at Planck scale is very different from what the continuum picture suggests. As we argued in the Introduction, this basic fact may well be the essential reason why the perturbation theory fails.

3. The geometrical observables can be defined on the full phase space of the classical theory; one need not restrict oneself to the constraint surface. Similarly, in the quantum theory, the analogous operators have been defined on the kinematic Hilbert space $H_{o}$, before imposing quantum constraints. The results are thus robust; they are not sensitive to the details of quantum dynamics. For example, they will continue to hold also in supergravity, and more generally, in any theory in which the triads and connections feature as a basic canonical pair.

4. There is, however, a subtlety. To see this, recall a basic difference between quantum mechanics and quantum field theory. In quantum mechanics, kinematics and dynamics are largely decoupled in the sense the same representation of the canonical commutation relations supports all interesting Hamiltonians. In quantum 
field theory, on the other hand, kinematics and dynamics are always weakly coupled: there are infinitely many inequivalent representations of the canonical commutation relations and, in general, different Hamiltonians are meaningful on different representations. In the present case, then, the key question is whether the quantum constraints of a given theory can be meaningfully imposed on $H_{o}$. Results to date indicate (but do not yet prove) that this is likely to be the case for constraints of general relativity. My general expectation is that this will also be the case for a class of theories such as supergravity which are "near general relativity" as far as geometry is concerned. What we have here is a glimpse into the nature of quantum geometry underlying this class of quantum gravity theories.

5. Note that $\hat{A}(S)$ and $\hat{V}(R)$ have been defined for any surface $S$ and any region $\mathrm{R}$ in $\Sigma$ and will therefore not be Dirac observables; they are not expected to commute with the quantum constraints. To obtain Dirac observables, one would have to specify $S$ and $R$ intrinsically. (For example, if we had only the Gauss and the diffeomorphism constraints, the total volume of $\Sigma$ would be a Dirac observable.) A natural strategy is to specify $S$ and $R$ using matter fields (see, e.g.,[31]). In view of the Hamiltonian constraint, the problem of providing an explicit specification of this type is extremely difficult. However, if a surface $S$ or a region $R$ were specified in this manner, Eqs (6) and (7) will provide the expressions of the associated area and volume operators.

6. Note that the expressions of the operators do not involve any free renormalization constants; they are completely unambiguous. For the two operators considered here, this is just a fact of calculations. However, there exist heuristic arguments that indicate that, in a more general context, if the end result of such a regularization procedure is an operator which has no background dependence, then these final operators should be free of arbitrary constants [10]. This reasoning is borne out in the construction of another geometric operator, associated with the classical observable $E(\omega):=\int d^{3} x\left(E_{i}^{a} E^{b i} \omega_{a} \omega_{b}\right)^{\frac{1}{2}}$, where $\omega$ is a smooth 1-form of compact support on $\Sigma$. (As in the case of area and volume, a square-root is essential to obtain a density of weight one which one can then integrate without reference to any background metric or volume element. Note incidentally that, since $\omega_{a}$ is arbitrary, classically, the functional $E(\omega)$ has the full information about the metric.) It is not known if there are other well-defined geometrical operators, e.g. one which carries information about the length functional directly.

\section{Quantum Einstein Equations}

We now turn to quantum dynamics. In the canonical approach, this is captured in the quantum constraints. Our task then is the following: i) Write the regulated constraints as operators on the kinematic Hilbert space $H_{o}$; ii) Check for anomalies; iii) If there are none, "solve" the quantum constraints; and, iv) On the space of solutions, introduce an appropriate Hilbert space structure. If all this can be achieved, one would have a coherent mathematical framework; one would say that quantum general relativity does exist non-perturbatively. One would, of course, still have to 
devise suitable approximation schemes to extract physical predictions.

There is however a key difficulty, of quite a general sort, associated with quantum constraints that needs to be resolved before we proceed further. To illustrate it, let us consider the example of a free relativistic particle in Minkowski space. Then, the classical configuration space is the 4-dimensional Minkowski space-time, the phase space is the cotangent bundle over it and there is a single dynamical constraint $C(x, p):=\eta^{a b} p_{a} p_{b}+m^{2}=0$. In quantum theory then, we can take $H_{o}:=L^{2}\left(R^{4}, d^{4} x\right)$ to be the fiducial, kinematical Hilbert space. The constraint can be promoted to a selfadjoint operator $\hat{C}:=-\left(\eta^{a b} \partial_{a} \partial_{b}-\mu^{2}\right)$ on $H_{o}$ (where $\left.\mu=m / \hbar\right)$. Thus solutions $\Psi(x)$ to the quantum constraint are easily obtained: they solve the Klein-Gordon equation. The problem is that none of the non-zero solutions lies in the kinematical Hilbert space $H_{o}$. (This is obvious in the momentum representation where the solutions $\psi(p)$ are distributions $\psi(p)=g(p) \delta\left(p \cdot p+m^{2}\right)$ for some well-behaved function $g(p)$ and therefore not square-integrable in the $L^{2}$-norm.) This is a rather general problem; it is in the exceptional case when the group generated by the constraint functions is compact that the solutions $\Psi$-i.e., physical states- belong to $H_{o}$. Thus, in the generic situation, we face two problems: What is the "home" of the physical quantum states? Once the home has been found and space of suitable solutions isolated, how is one to introduce an inner product on this space?

Fortunately, there exists [32] a general approach to both these problem, based on the idea of "averaging over" the orbit of the group generated by the constraints. In the present case, one first considers the 1-parameter group of unitary transformations, $U(\lambda):=\exp i \lambda \hat{C}$, generated by the constraint $\hat{C}$. The idea is to construct solutions by averaging suitable elements of $H_{o}$ over the 1-parameter group generated by $U(\lambda)$. For the result to be manageable, however, we have to restrict the initial element to a "nice" (dense) subspace $\mathcal{S}$ of $H_{o}$. Let us choose this $\mathcal{S}$ to be the space of smooth test functions of compact support. Then, given any test field $f$, one can show that $\Psi_{f}:=\int d \lambda U(\lambda) \circ f$ exists as a well-defined distribution over $\mathcal{S}$ and solves the quantum constraint. Thus, the natural home for these solutions is $\mathcal{S}^{\star}$, the topological dual to $\mathcal{S}$ we began with. Furthermore, we can now naturally define an inner product: $\left(\Psi_{f}, \Psi_{h}\right):=\Psi_{f}(h) \equiv \int d^{4} x \bar{\Psi}_{f}(x) h(x)$. It turns out that this is the correct inner product. Thus, in this example, the group averaging procedure provides an answer to both our questions. (As far as I know, however, there are no general theorems to ensure a priori that the method is so directly applicable in more general situations. The practical strategy is to apply it to any given case and see if the final inner-product is well-defined, i.e., positive definite.)

The procedure is applicable to the vector (or the diffeomorphism) constraint of general relativity [23]. One first promotes the formal "exponentiated forms" of diffeomorphism constraints to well-defined, unitary operators on the kinematical Hilbert space $H_{o}$. (These operators are unitary because the measure $\mu_{o}$ is diffeomorphism invariant.) In the second step, one checks for anomalies. In various lattice regularizations, these were encountered essentially because the lattice regularization manifestly breaks the diffeomorphism invariance. Here, we are working directly in the continuum, have a well-defined Hilbert space and unitary operators 
$U_{\vec{N}}(\lambda)$ thereon, for each analytic vector field $\vec{N}$ on $\Sigma$. We can directly check their algebra. One finds that there are no anomalies. One can now proceed to the last two steps: solving the constraint and introducing the inner product on the space of physical states. Here we apply the group averaging technique. As one might expect, the role of the dense sub-space $\mathcal{S}$ is played by the space of cylindrical functions $\operatorname{Cyl}(\overline{\mathcal{A} / \mathcal{G}})$ and the physical states lie in its topological dual. They are not normalizable with respect to $H_{o}$. Nonetheless, using the procedure outlined above, one can endow them with an inner product and obtain a Hilbert space $H_{d}$ of solutions to the diffeomorphism constraints ${ }^{5}$. Roughly, states in $H_{d}$ can be labelled by equivalence classes of spin networks, where two are regarded as equivalent if they are related by the action of the diffeomorphism group. This provides a precise formulation of the interplay between knot theory and general relativity that was anticipated by Rovelli and Smolin already in [7] and also brings out a number of subtleties. Finally, the inner-product on $H_{d}$ automatically incorporates the "reality conditions" $[8,33]$ correctly. More precisely, the projection to $H_{d}$ of operators on $H_{o}$ which are self-adjoint and commute with the diffeomorphism constraints are guaranteed to be self-adjoint on $H_{d}$.

The Gauss constraint is already taken care of since we are working on the space $\overline{\mathcal{A} / \mathcal{G}}$ of gauge equivalent (generalized) connections. Alternatively, as indicated in [23], one could first begin with the space $\overline{\mathcal{A}}$ of (generalized) connections, show that there are no anomalies, impose the Gauss constraint using group averaging, and show that the resulting Hilbert space of states is precisely the space $H_{o}$ that we began with in this paper.

Finally, we come to the difficult Hamiltonian constraint, the analog of the Wheeler-DeWitt equation. There has been considerable work on solutions to this constraint (see, e.g., [34-37]) and several fascinating results have been obtained. For example, some of the solutions have been identified with well-known knot invariants. However, most of this work is of a rather formal nature; for example, it is generally unclear if the underlying theory is Euclidean or Lorentzian. In keeping with the general spirit of this review, here, I will set these results aside and consider instead a more recent development which focuses more on the structural issues rather than on specific solutions.

Let us begin with the third constraint in Eq (3), even though it refers to the Euclidean -rather than the Lorentzian- theory. The task is to define the corresponding quantum constraint operators on the Hilbert space $H_{d}$ of solutions to the diffeomorphism constraints. Using a key idea due to Rovelli and Smolin [38], a regulated form of this operator has been constructed and it has been shown that, in the appropriate sense, the constraint algebra closes without anomalies [39]. While this progress is notable, I should point out that, in contrast to the regularization

\footnotetext{
5 In the analytic category used in this paper, we have been able to obtain an infinite dimensional family of solutions but it is not clear if this class exhausts all physically interesting situations. This problem would disappear if we could extend the entire discussion to the physically better suited smooth category along the lines initiated by Baez and Sawin [15]. For details, see [23].
} 
of geometrical operators or the imposition of the diffeomorphism constraint, these calculations should be regarded as preliminary explorations. Specifically, there is considerable freedom at an intermediate step and no compelling reason to adopt the specific prescription used there. Furthermore, properties of the resulting constraint operator have not been investigated in detail. Even heuristically, it does not appear to be self-adjoint. On the one hand, as Kuchař [40] has pointed out, this would consistent with the closure of the constraint algebra since in finite dimensional models with an analogous algebra, the constraints would not close if the analog of the Hamiltonian constraint were self-adjoint (although this possibility is not ruled out in infinite dimensions due to subtleties associated with regularization.) However, non self-adjointness would also make it impossible to use the group averaging method at least directly. In spite of these important limitations, the calculation does represent a first serious attempt to regularize the Hamiltonian constraint rigorously and therefore holds considerable promise. In geometrodynamics, for example, a comparable stage is yet to be reached with respect to the Wheeler-DeWitt equation.

There is, however, a much more serious difficulty: The classical constraint we began with refers to the Euclidean signature while, physically, we need to solve the Lorentzian constraint. Thus, we need a generalized Wick transformation which will map the solutions to the quantum Euclidean constraint to those of the Lorentzian. At first, the problem looks hopelessly difficult. However, recently, Thiemann [41] has suggested a strategy in the context of a "coherent state transform" that will map complex-valued functions of real $S U(2)$ connections to holomorphic functions of an $S L(2, C)$ connection. The same strategy can be adopted in a purely real formulation used in this report (and also extended to allow for the presence of matter sources) [42]. To see how this works, let us return to the classical theory, where the phase space, canonical variables and constraints are all real in both signatures. The idea is to define an automorphism $W$ on the algebra of complex-valued functions on the phase space -i.e., a map which preserves linear combinations, products and Poisson brackets of functions- which maps the Euclidean Hamiltonian constraint functional (3a) to the Lorentzian one (3b). $W$ is generated by a phase-space function $T$ as follows:

$$
W \circ f:=f+\{T, f\}+\frac{1}{2 !}\{T,\{T, f\}\}+\ldots \equiv \sum_{i=0}^{\infty} \frac{1}{n !}\{T, f\}_{n},
$$

where $\{T, f\}$ is the Poisson bracket between $T$ and $f$ and $\{T, f\}_{n}$, the repeated Poisson bracket of $n T$-factors with $f$ and the generating function $T$ is given by:

$$
T:=\frac{i \pi}{2} \int_{\Sigma} d^{3} x K_{a}^{i} E_{i}^{a} \equiv \frac{i \pi}{2}\left\{V, H_{E}\right\} .
$$

Here, $V$ denotes the total volume functional and $H_{E}:=\int d^{3} x g^{-\frac{1}{2}} \mathcal{S}$, where, as before, $\mathcal{S}$ is the Euclidean scalar or Hamiltonian constraint (see Eq (1.3). Note that $T$ is just the integral of the trace of the extrinsic curvature over $\Sigma$.) It is straightforward to verify that $W \circ \mathcal{S}=\mathcal{S}_{L}$. Hence, if we could promote $T$ to a quantum operator, we would have the generalized Wick transform $\hat{W}:=\exp -\hat{T} / i \hbar$ in the quantum theory which would map the solutions to the Euclidean Hamiltonian constraint to those of the Lorentzian: We would have

$$
\hat{\mathcal{S}} \circ \Psi=0 \quad \Longrightarrow \quad \hat{\mathcal{S}}_{L} \circ(\hat{W} \circ \Psi)=0
$$


$\hat{\mathcal{S}}_{L}$ being the Lorentzian Hamiltonian constraint. That is, while we did not have, a priori, a way of defining the Lorentzian Hamiltonian constraint operator, since classically $W$ maps the Euclidean Hamiltonian constraint functional to the Lorentzian one, we can simply define the Lorentzian quantum constraint $\hat{\mathcal{S}}_{L}$ by $\hat{\mathcal{S}}_{L}=\hat{W} \circ \hat{\mathcal{S}} \circ \hat{W}^{-1}$. This is an attractive strategy especially since, as Eq (10) shows, $\hat{T}$ could be constructed from the total volume operator (on which we have full control) and the integrated Euclidean Hamiltonian constraint.

To summarize, if the Euclidean quantum constraint $\hat{\mathcal{S}}$ can be regularized in a more satisfactory fashion (so as to be free of the drawbacks of the present scheme discussed above) and the operator $\hat{T}$ defined and shown to have certain properties on $H_{d}$, we would conclude that all quantum constraints of general relativity can be imposed consistently, i.e., that quantum general relativity is consistent at a nonperturbative level. Note that it is not essential to exhibit the general solution to the Hamiltonian constraint; indeed, even in the classical case, we do not have a general solution to the Einstein equations. As in the classical theory, what we need is a few simple solutions which can be interpreted and a degree of control on the structure of the space of solutions.

We will conclude with a few remarks on the generalized Wick transformation. First, note that $W$ does not arise from a canonical transformation on the real phase space: the generating function $T$ is imaginary, rather than real. (Thus, in quantum theory, $\hat{W}$ is not expected to preserve norms.) As far as I can tell, even on the complex phase space, the canonical transformation generated by $T$ does not have a simple geometric property which can readily enable one to interpret it directly as a "Wick rotation" from the Euclidean phase space to the Lorentzian. In particular, given a specific solution to the classical Euclidean constraint, $W$ does not provide us with a solution to the Lorentzian constraint. It is a well-defined mapping on the space of functions on the phase space, rather than on the (real) phase space itself. Furthermore, in general, it maps real functions to complex-valued functions; the Hamiltonian constraint is more of an exception than a rule where a real function is mapped to another real function. (For example, while $f(x) \mapsto f(i x)$ will in general map real functions to complex, $\cos x$ is again mapped to a real function, $\cosh x$.) Next, the action of $W$ does preserve the vector and the Gauss constraints (modulo overall constants) so that if, as in the construction sketched above, $\Psi$ were to satisfy all Euclidean constraints, $\hat{W} \circ \Psi$ would satisfy all Lorentzian constraints. Finally, one can ask whether, on the phase space, $W$ maps the Euclidean action to the Lorentzian. This is indeed the case if one simultaneously transforms lapses and shifts via $\left(\underline{N}, N^{a}\right) \mapsto\left(-\underline{N}, N^{a}\right)$, where $\underline{N}$ is the lapse field with density weight -1 . These transformation properties of lapses and shifts are, however, different from those one encounters in quantum cosmology.

\section{Discussion}

The new, background independent functional calculus on the quantum configuration space $\overline{\mathcal{A} / \mathcal{G}}$ has enabled us to develop the quantization program systematically. 
The level of mathematical precision is such that all underlying assumptions are explicit and we can be confident that there are no hidden infinities. I would like to emphasize that, for the problem under consideration, this degree of precision is not a luxury; it is essential. Let me elaborate on this point since it is often overlooked. In theoretical physics in general, and in quantum field theory in particular, we often do formal manipulations, subtract infinities and extract physical answers. We do not worry about defining measures rigorously and are content even if the perturbation series we arrive at diverges uncontrollably so long as individual terms in the series are finite. In quantum gravity, however, I believe that we can not afford to be so cavalier. For, the central problem is somewhat different now. In the case of other three interactions, we have piles of experimental data and the central task is that of organizing it in a coherent fashion and making further predictions that can be tested by experiments. In quantum gravity, we are not blessed with this richness. At the present stage, the key problem is that of consistency: Can the principles of general relativity and quantum theory be unified in a mathematically consistent fashion? To be confident of the answer, we are forced to elevate our mathematical standards. Indeed, perturbative treatments have taught us that it would be extremely unwise to be satisfied with formal manipulations.

The techniques underlying our background-independent functional calculus may seem somewhat unfamiliar to most physicists. The overall situation is rather analogous to the introduction, in the sixties, of global techniques in general relativity. Until then, most relativists were content with local calculations and worked exclusively with coordinates. When the various notions of causality and even the definitions of a singularity were first introduced, they seemed exotic to the practitioners in the field as they were outside of what was then the mainstream. The new techniques did not invalidate the use of coordinate methods for local problems. However, they turned out to be indispensable to the analysis of global issues associated, e.g., with horizons and singularities which were then emerging as the frontier problems. These were the types of problems that the older methods could not handle. Indeed, using local methods, one could not even define the notion of the event horizon of a black-hole much less prove theorems about their properties. I believe that the situation with the new functional calculus is quite analogous. These techniques are essential to handle the question of mathematical consistency, the frontier issue at the present stage of our understanding of quantum gravity. Traditional tools, powerful as they are in the analysis of other interactions, appear to be insufficient to face this issue non-perturbatively, without any recourse to a background geometry. With the new calculus, we have tools to meet this challenge.

As we saw, these techniques have already been applied to two problems with considerable success: probing quantum geometry and solving the quantum constraints of the general relativity.

The picture of the geometry at the Planck scale that emerged turned out to be very different from the continuum image. In particular, operators representing areas of 2-surfaces and volumes of 3-dimensional regions have discrete spectra. This departure from the continuum -and particularly the discreteness of the spectrum of 
the area operators-may well hold the key to some of the current puzzles such as the relation between statistical entropy and the area of black hole horizons [43]. The fundamental, Planck scale excitations of the gravitational field are 1-dimensional and the corresponding geometry is distributional. As was pointed out in Section 3, in this respect, there is a close similarity with polymer physics. Although polymers and the basic phonon excitations in them are 1-dimensional, in suitably complex configurations, they exhibit 3-dimensional properties. The same is true of geometry $[3,44]$ : continuum 3-dimensional geometries do arise, but as approximations. More precisely, the Hilbert space $H_{o}$ admits states which can be interpreted as "semiclassical" in the sense that, when coarse-grained using a macroscopic length scale, they become indistinguishable from smooth continuum geometries in three dimensions. For example, given a flat 3-metric $g_{a b}^{o}$ on $R^{3}$, and a macroscopic length scale $L$, we can ask for states $\Psi$ in $H_{o}$ which have the property that for all regions with $g^{\circ}$-volume of the order of $L^{3}$ or bigger and for all surfaces $S$ which are slowly varying on the scale $L$, the eigenvalues of the volume and area operator approximate the values one would obtain classically using $g_{a b}^{o}$, up to corrections of the order $O\left(\ell_{P} / L\right)$. Such states exist and can be constructed using techniques from "statistical geometry" that underlie random lattices [44]. These states have been generically called "weaves" because they tell us how to weave a classical geometry from "quantum threads" -the elementary excitations of geometry.

There is an indirect test of these ideas. Although one expects the laboratory physics to be insensitive to the detailed predictions of quantum gravity, it may well be that to do this physics in a coherent fashion, one has to supplement the standard description with some qualitative ideas from quantum gravity ${ }^{6}$. For instance, in Minkowskian quantum field theories, the main difficulty comes from the ultraviolet divergences which arise because we integrate over arbitrarily large momenta, i.e., arbitrarily short distances. A more accurate representation of the underlying geometry is given by a weave state. So, rather than doing quantum field theory on a continuum, we should really do it on a weave state. Such a theory would be free of ultra-violet divergences. The key question is whether it reproduces the results of the standard perturbation theory at "low energy." Heuristically, one would expect this to be the case: Since the standard theories are renormalizable, their predictions for phenomena at the $10^{-17} \mathrm{~cm}$ scale should be insensitive to the details of the microstructure of weave states at the Planck scale. (Recall that the weave geometry differs from the continuum only up to terms $O\left(\ell_{P} / L\right) \approx\left(10^{-33} / 10^{-17}\right) \approx 10^{-16}$.) If one could show this result in detail -i.e., establish that the predictions of a finite quantum field theory on a weave state agree with those of the standard perturbation

\footnotetext{
6 There are numerous examples of such situations in other branches of physics. For example, in astrophysics, one can generally work with Newtonian gravity. However, to discuss the density distributions of stars near the centers of galaxies such as M87 which have large black holes in their centers, one simply changes the boundary conditions on Newtonian equations, allowing stars to disappear once they cross the event horizons. Thus, although the use full general relativity is unnecessary, some "qualitatively new" features of this more accurate theory have to be incorporated.
} 
theory for laboratory energies- one could take the rich phenomenological data from particle physics as an indirect evidence for the discrete structure of the Planck scale geometry.

The second main application of the framework is to quantum Einstein equations. The diffeomorphism constraints could be regulated on the kinematical Hilbert space $H_{o}$ in a manner that is anomaly-free and the space of solutions could be given a Hilbert space structure using the group averaging technique. There is also progress on the Hamiltonian constraint and the idea of using a generalized Wick transformation is tantalizing. However, more work is needed to make these results definitive. It is quite possible that to obtain a satisfactory regularization, one would have to bring in the notion of "framed networks" and replace the gauge group $S U(2)$ by its "quantum version". However, if these last problems could be resolved, one would conclude that quantum general relativity does exist non-perturbatively. It is already clear that its structure would be very different from that envisaged in perturbative treatments which are all rooted in a continuum picture. My own view is that there may well exist several inequivalent ways of regulating the Hamiltonian constraint, leading to inequivalent theories. Indeed, this is likely to be physically the most important source of non-uniqueness of the quantum theory. If the theory does admit such inequivalent sectors, it would be all the more important to develop approximation schemes to extract their physical content. They will be motivated by the structure of the exact theory and therefore likely to be quite different from the ones used in the standard perturbative treatments. Work is in progress along several lines in this area.

An attractive possibility is to combine the strengths of string theory and the nonperturbative approach discussed here. String theory provides a "tight" strategy to couple matter for which no analogous principle is known within general relativity. On the other hand, at least in practice, string theory is essentially perturbative and needs a background continuum geometry. Quantum general relativity needs no background fields and provides a specific picture of quantum geometry. A natural strategy then would be to investigate strings on these quantum geometries. Indeed, since the excitations of geometry are along 1-dimensional graphs, it it natural to incorporate matter through strings winding around loops in these graphs. This might provide for us a viable perturbation theory. Results of Klebanov and Susskind [45] suggest a concrete direction for this work.

To conclude, let me address an obvious question about the new functional calculus: Since it refers to theories of connections, can it not be used for a nonperturbative treatment of Yang-Mills theories? Unfortunately, in the general case, there is an obstruction: While our emphasis has been on diffeomorphism invariance and absence of background fields, Yang-Mills theories depend on the background Minkowskian metric rather heavily. Thus, to make the framework directly amenable to general Yang-Mills theories, we would have to develop it further in a substantial way by adding new techniques which are tailored to the presence of a flat background metric. There is, however, an exception: two space-time dimensions where one only needs an area element, rather than a metric, to specify the Yang-Mills action. Thus, 
the theories are now invariant under area preserving diffeomorphisms. In this case, our techniques are directly applicable and have led to a number of new results [46]. These include: explicit expressions of the Schwinger functions for Wilson loops and a direct proof of the equivalence of the Euclidean path integral formulation and the Hamiltonian quantum theory. Furthermore, unlike in other approaches, the invariance of the quantum theory under area preserving diffeomorphisms is manifest in this treatment.

\section{Acknowledgements}

Most of the recent results reported here were obtained in collaboration with $\mathrm{C}$. Isham, D. Marolf, J. Mourão, T. Thiemann and, especially, J. Lewandowski. I am most grateful to them for constant intellectual stimulation over the past two years and for their patience with my slow pace in writing up the results. I am grateful also to John Baez, Rodolfo Gambini, Karel Kuchař, Jorge Pullin, Carlo Rovelli, and Lee Smolin for their numerous suggestions, comments and criticisms which were often vital. This work was supported in part by the NSF grant PHY 93-96246 and by the Eberly Research Funds of the Penn State University.

\section{References}

1. P. Faria de Veiga, Ecole Polytechnique thesis (1990); C. de Calan, P. Faria de Veiga, J. Magnen and R. Sénéor, Phy. Rev. Lett. 66 (1991) 3233; A. S. Wightman, in: Mathematical Physics Towards XXIst Century, eds R. N. Sen and A. Gersten (Ben Gurion University Press, 1994).

2. D. Amati, M. Ciafolini and G. Veneziano, Nucl. Phys. B347 (1990) 550.

3. A. Ashtekar, C. Rovelli and L. Smolin, Phys. Rev. Lett. 69 (1992) 237;

4. A. Agishtein and A. Migdal, Mod. Phys. Lett. 7 (1992) 85.

5. J. Iwasaki and C. Rovelli, Int. J. Mod. Phys. D1 (1993) 533; Class. \& Quantum Grav.11 (1994) 1653.

6. T. Jacobson and L. Smolin, Nucl. Phys. B299 (1988) 295.

7. C. Rovelli and L. Smolin, Phys. Rev. Lett. 72 (1994) 446.

8. A. Ashtekar, Non-Perturbative Canonical Gravity (World Scientific, Singapore, 1991); in Gravitation and Quantization eds B. Julia and j. Zinn-Justin (Elsevier, Amsterdam 1995).

9. R. Rovelli, Class. \& Quantum Grav. 8 (1991) 1613.

10. L. Smolin, in Quantum Gravity and Cosmology eds J. P. Mercader, H. Solà and E. Verdaguer (World Scientific, Singapore, 1992).

11. R. Gambini and J. Pullin, Loops, Knots, Gauge Theories and Quantum Gravity (Cambridge University Press, Cambridge, 1996).

12. A. Ashtekar, Phys. Rev. Lett. 57 (1986) 2244; Phys. Rev.D36 (1987) 1587.

13. A. Ashtekar, in Mathematics and General Relativity (AMS, Providence, 1987), J.F. Barbero G. Phys. Rev D51 (1995) 5507.

14. A. Ashtekar and C. J. Isham, Class. E Quantum Grav. 9 (1992) 1433. 
15. J. C. Baez and S. Sawin, Functional integration of spaces of connections, q-alg/9507023.

16. A. Rendall, Class. \& Quantum Grav. 10 (1993) 605.

17. A. Ashtekar and J. Lewandowski, in Knots and Quantum Gravity, ed J. Baez (Oxford University Press, Oxford, 1994).

18. J. Baez, Lett. Math. Phys.31 (1994) 213; in The Proceedings of the Conference on Quantum Topology, ed D. N. Yetter (World Scientific, Singapore, in press).

19. D. Marolf and J. Morão, Commun. Math. Phys. 170 (1995) 583.

20. A. Ashtekar and J. Lewandowski, J. Math. Phys. 36 (1995) 2170.

21. A. Ashtekar and J. Lewandowski, J. Geo. \& Phys. 17 (1995) 191.

22. A. Ashtekar, J. Lewandowski, D. Marolf, J. Mourão and T. Thiemann, Coherent state transform on the space of connections, J. Funct. Analysis (in press).

23. A. Ashtekar, J. Lewandowski, D. Marolf, J. Mourão and T. Thiemann, J. Math. Phys.36 (1995) 6456.

24. J. C. Baez, Spin networks in gauge theory, Adv. Math. (in press); Spin networks in non-perturbative quantum gravity, gr-qc/9504036.

25. L. Smolin (private communication).

26. C. Rovelli and L. Smolin, Spin networks and quantum gravity, preprint CGPG-95/4-1.

27. R. Penrose, in Quantum Theory and Beyond, ed T. Bastin, Cambridge University Press, Cambridge 1971).

28. A. Ashtekar and J. Lewandowski, Quantum geometry, (preprint).

29. C. Rovelli and L. Smolin, Nucl. Phys.B442, 593 (1995).

30. R. Loll, The volume operator in discretized gravity, (pre-print).

31. C. Rovelli, Nucl. Phys.B405 (1993) 797; L. Smolin, Phys. Rev.D49 (1994) 4028.

32. A. Higuchi, Class. \& Quantum Grav.8 (1991) 1983; 2023; N. P. Landsman J. Geo. \& Phys.15 (1995) 285.

33. A. Ashtekar and R. S. Tate, J. Math. Phys. 34 (1994) 6434.

34. B. Brügmann and J. Pullin, Nucl. Phys. B363 (1991) 221; B390 (1993) 399.

35. B. Brügmann, R. Gambini and J. Pullin, Phys. Rev. Lett. 68 (1992) 431.

36. H. Nicolai and H. J. Matschull, J. Geo. and Phys. 11 (1993) 15; H. J. Matschull, pre-print gr/qc 9305025;

37. H. A. Morales-Técotl and C. Rovelli, Phys. Rev. Lett.72 (1994) 3642.

38. C. Rovelli and L. Smolin, (private communication).

39. A. Ashtekar and J. Lewandowski (in preparation).

40. K. Kuchař (private communication).

41. T. Thiemann, Reality conditions inducing transforms for quantum gauge fields and quantum gravity, (pre-print).

42. A. Ashtekar, A generalized Wick transform for gravity, (pre-print). 
43. J. D. Bekenstein and V. F. Mukhanov, Spectroscopy of quantum black holes, (preprint).

44. A. Ashtekar and L. Bombelli (in preparation).

45. I. Klebanov and L. Susskind, Nucl. Phys. B309 (1988) 175.

46. A. Ashtekar, J. Lewandowski, D. Marolf, J. Moraõ and T. Thiemann, in Geometry of Constrained Dynamical Systems, ed. J. Charap (Cambridge University Press, Cambridge, 1994); Quantum Yang-Mills theory in two dimensions: A complete solution, (pre-print). 